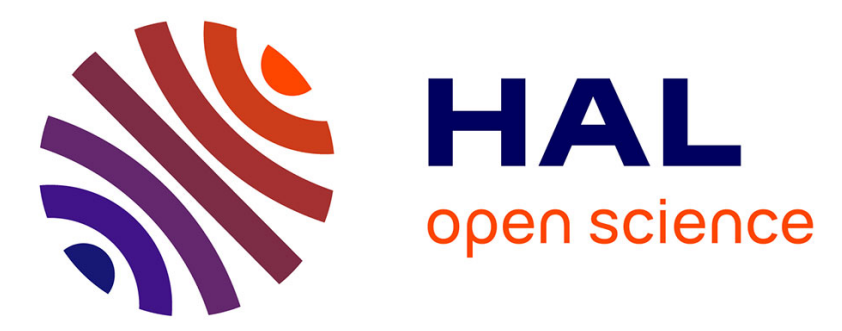

\title{
Different phenotypic expression of KPC $\beta$-lactamase variants and challenges in their detection
}

Saoussen Oueslati, Linda Tlili, Cynthia Exilie, Sandrine Bernabeu, Bogdan Iorga, Rémy Bonnin, Laurent Dortet, Thierry Naas

\section{- To cite this version:}

Saoussen Oueslati, Linda Tlili, Cynthia Exilie, Sandrine Bernabeu, Bogdan Iorga, et al.. Different phenotypic expression of KPC $\beta$-lactamase variants and challenges in their detection. Journal of Antimicrobial Chemotherapy, 2020, 75 (3), pp.769-771. 10.1093/jac/dkz508 . hal-02840859

\section{HAL Id: hal-02840859 \\ https://hal.science/hal-02840859}

Submitted on 7 Dec 2020

HAL is a multi-disciplinary open access archive for the deposit and dissemination of scientific research documents, whether they are published or not. The documents may come from teaching and research institutions in France or abroad, or from public or private research centers.
L'archive ouverte pluridisciplinaire HAL, est destinée au dépôt et à la diffusion de documents scientifiques de niveau recherche, publiés ou non, émanant des établissements d'enseignement et de recherche français ou étrangers, des laboratoires publics ou privés. 


\section{challenges in their detection}

${ }^{1}$ EA7361 "Structure, dynamic, function and expression of broad spectrum $\beta$-lactamases", Faculty of Medicine

${ }^{2}$ Bacteriology-Hygiene unit, Assistance Publique/Hôpitaux de Paris, Bicêtre Hospital, Le Kremlin-Bicêtre, France

$11{ }^{3}$ Institut de Chimie des Substances Naturelles, CNRS UPR 2301, Université Paris-Saclay, Labex LERMIT, Gif-sur-

12 Yvette, France.

$13 \quad{ }^{4}$ Associated French National Reference Center for Antibiotic Resistance: Carbapenemase-producing

14 Enterobacteriaceae, Le Kremlin-Bicêtre, France

15

16 Running title: Phenotypic expressions of KPC variants (38 characters spaces included)

17 Words : 1059

18 Table: 1 ; Supplemental Figure S1

19 *Corresponding author: Service de Bactériologie-Hygiène, Hôpital de Bicêtre 78 rue du Général Leclerc, 94275

20 Le Kremlin-Bicêtre, France.

21 Tel : +33145212019;

22 Fax : +33 1452163 40,

23 thierry.naas@aphp.fr 
Carbapenem-resistance among Enterobacteriaceae has become a major public health

issue owing to their rapid worldwide spread. ${ }^{1}$ In Enterobacteriaceae this resistance is largely due to the production of carbapenemases, the most prevalent throughout the world being KPC-, VIM-, IMP-, NDM- and OXA-48 type variants. KPC-2, initially described in the USA in $2001,^{2}$ went global in less than a decade likely as a result of being located on a transposon, Tn4401 capable of high frequency transposition, itself inserted on self-conjugative plasmids, and its frequent association with the highly successful K. pneumoniae clonal group $258 .^{3}$ Today, more than 44 natural KPC variants have been described, but only a few of them have been characterized in terms of $\beta$-lactam hydrolytic properties. ${ }^{4}$ It is crucial to understand the impact of single amino-acid changes on the hydrolytic profile of KPC-variants and the ability of commercially-available diagnostic tests to efficiently detect them.

In this work, we have compared the phenotypic expressions of 14 KPC variants either natural or generated by site-directed mutagenesis and evaluated the performance of several diagnostic tests to detect them. Bla $a_{\mathrm{KPC}-2}$ and $b / a_{\mathrm{KPC}-3}$ alleles were cloned into pTOPO vector along with its natural ribosomal binding site (RBS) upstream, and electroporated into electrocompetent $E$. coli TOP10 as previously described. ${ }^{5}$ The resulting plasmids were used to construct 11 single $b / a_{\mathrm{KPC}-2}$ mutants and one single $b / a_{\mathrm{KPC}-3}$ mutant using the QuikChange II SiteDirected Mutagenesis Kit (Agilent Technologies, Les Ulis, France) (Table 1). Thus among the 12 generated mutants (Figure S1A), four were natural variants of KPC-2 [KPC-5 (P104R) ${ }^{6}$, KPC6 (V240G)7, KPC-12 (L168M), and KPC-33 (D179Y)] and one of KPC-3 [KPC-31 (D179Y)]. Six substitutions correspond to natural substitutions that occurred in KPC-2, but that were not reported alone: V240A, G147K, A120L, D92G, W105A and W105G. Finally, the last mutant has a substitution C238S, which is a conserved residue that establishes a di-sulfide bridge with the 
C68. MICs were performed using the Etest (bioMérieux, Marcy-I’Etoile, France) and detection tests were performed according to manufacturers' recommendations. Phenotypic profiles and diagnostic testing results are summarized in Table 1. Increased MICs for aztreonam and cephalosporins without any modification of those for carbapenems have been observed with mutants in position 240. On the opposite substitutions at positions 168 and 92 led to low carbapenem MICs, but yet detectable carbapenem hydrolysis (as evidenced using biochemical tests, see Table 1). The mutants P104R and G147K led to increased MICs for aztreonam and cephalosporins, with slight decreased MICs for carbapenems. The mutations on residues 105 and 238 resulted in a global decrease in MICs for all $\beta$-lactams except amoxicillin, with a stronger impact observed for the C238S mutant. With all of these mutants (including those with highly increased MICs for ceftazidime, such as P104R, V240G and V240A) avibactam was able to restore ceftazidime susceptibility. Interestingly, KPC-31 and KPC-33 with a D179Y substitution, exhibit reduced MICs for all $\beta$-lactams, including aztreonam and amoxicillin, except for ceftazidime, for which increased MICs were observed. In addition, these two mutants led to MICs for the combination of ceftazidime/avibactam, that were limit to resistance according to CLSI and EUCAST. These results suggest that avibactam is not capable of inhibiting KPC-31 and KPC-33 efficiently. In order to confirm this hypothesis IC50s were measured with purified proteins. ${ }^{5}$ The IC50 values for KPC-31 $(20 \mu \mathrm{M})$ and KPC-33 $(42 \mu \mathrm{M})$ were 100 fold higher than those of KPC-2 (230nM) and KPC-3 (350nM), further supporting that the residue D179 is important in the binding of avibactam..$^{8,9,5}$

Overall, all tested residues, except A120, have a direct impact on the phenotypic expression profile of KPC enzymes. Residues 168 and 179 are located on the $\Omega$ loop, which is known to play a crucial role in the substrate selectivity of the enzyme. ${ }^{9}$ Residues 105,104 and 240 delimit the cavity of the active site. Accordingly, hydrolysis spectrum is influenced by the 
nature of the residues at these positions. Since the two cysteines C238 and C68 (adjacent to the S70) form a disulfide bridge that stabilizes the KPC folding, it was expected to identify a dramatic impact of the C238S substitution on the catalytic activity of the enzyme, as shown for other class A carbapenemases such as SME and GES-5. ${ }^{10,11}$ Finally, although located at the opposite of the active site, residues 92 and 147 have also a crucial impact on KPC activity. Molecular modeling of the G147K mutant, performed with UCSF Chimera software, ${ }^{12}$ shows a clash with G143 located on the helix $\alpha 6$, which is in vicinity of the active site (Figure S1B). This clash might lead to a switch of this helix that subsequently impact on the shape of the active site.

Several diagnostic tests based on imipenem hydrolysis (biochemical and MALDITOF-based), Lateral flow immunoassays and molecular techniques (PCR) were evaluated on these different mutants. Unlike molecular tests that were able to detect all mutants, biochemical tests based on imipenem hydrolysi: Carba NP test ${ }^{13}$, Rapidec CARBA NP ${ }^{13}$ (bioMérieux, Marcy l'Etoile, France) and $\beta$-CARBA ${ }^{14}$ (BioRad, Marnes la Coquette, France), and MALDI-TOF -based MBT STAR-Carba IVD ${ }^{15}$ (Bruker, Illkirch-Graffenstaden, France) were able to distinguish between KPC variants with carbapenem hydrolytic capacities from those lacking any carbapenemase activity (Table 1). Molecular assays, being an in-house KPC specific PCR or a commercially-available PCR (Xpert Carba, Cepheid, Maurens-Scopont, France) detected accurately all variants. NG-test Carba5 (NG Biotech, Guipry, France) and Resist-4 O.K.N.V. KSet (Coris, Gembloux - Belgium) lateral flow immunoassays (LFIA) ${ }^{16}$ assays were also able to detect all KPC variants except KPC-31 and KPC-33. ${ }^{4}$ As the two latter variants lack carbapenemase activity, the non-detection does not lead to false negative results in terms of true carbapenemase detection. As these 2 variants lead to avibactam resistance, it would be nevertheless important that they are detected, but not classified as carbapenemases. 
97 Whether, carbapenems might be used to treat infections with KPC-31 or KPC-33-producers, is

98 still debatable, but according to EUCAST guidelines the answer is Yes, as the MICs for

99 imipenem are in the susceptibility range.

100 Overall, our results demonstrate that KPC variants are nor equal, probably selected by

101 different antibiotic usages. This is the case for KPC-5 that leads to high level cefixime

102 resistance. Some KPC variants cannot be considered as carbapenemases anymore. This is the

103 case for KPC-31 and KPC-33 that have lost their carbapenemase activity in a similar manner to

104 KPC-14 and KPC-28 variants. ${ }^{5}$ These peculiar variants are challenging for their detection, and

105 will require complementary approaches combining molecular or LFIA together with

106 carbapenem hydrolysis detection systems.

107

108 Funding

109 This work was supported by the Assistance Publique - Hôpitaux de Paris, by a grant from the

110 Université Paris-Sud (EA 7361), and by the LabEx LERMIT with a grant from the French National

111 Research Agency (ANR-10-LABX-33) and a grant from the French National Research Agency

112 (ANR-17-ASTR-0018).

113 Transparency declarations

114 LD is co-inventor of the Carba NP Test, which patent has been licensed to bioMérieux (La 115 Balmes les Grottes, France). All other authors: none to declare.

117 References

118 1. Eichenberger EM, Thaden JT. Epidemiology and Mechanisms of Resistance of Extensively

119 Drug Resistant Gram-Negative Bacteria. Antibiotics (Basel) 2019; 8(2). pii: E37. doi:

$120 \quad$ 10.3390/antibiotics8020037. 
121 2. Yigit $H$, Queenan AM, Anderson GJ, et al. Novel Carbapenem-Hydrolyzing $\beta$-Lactamase, KPC-

122 1, from a Carbapenem-Resistant Strain of Klebsiella pneumoniae. Antimicrob Agents $123 \quad$ Chemother 2001; 45: 1151-61.

124 3. Peirano G, Bradford PA, Kazmierczak KM, et al. Importance of Clonal Complex 258 and 125 IncFK2-like Plasmids among a Global Collection of Klebsiella pneumoniae with blaKPC. $126 \quad$ Antimicrob Agents Chemother 2017; 61: e02610-16.

127 4. Naas T, Oueslati S, Bonnin RA, et al. Beta-lactamase database (BLDB) - structure and function. Journal of Enzyme Inhibition and Medicinal Chemistry 2017; 32: 917-9.

5. Wolter DJ, Kurpiel PM, Woodford N, et al. Phenotypic and Enzymatic Comparative Analysis of the Novel KPC Variant KPC-5 and Its Evolutionary Variants, KPC-2 and KPC-4. Antimicrob Agents Chemother 2009; 53: 557-62.

6. Lamoureaux TL, Frase H, Antunes NT, et al. Antibiotic Resistance and Substrate Profiles of the Class A Carbapenemase KPC-6. Antimicrob Agents Chemother 2012; 56: 6006-8.

7. Oueslati S, lorga BI, Tlili L, et al. Unravelling ceftazidime/avibactam resistance of KPC-28, a KPC-2 variant lacking carbapenemase activity. J Antimicrob Chemother 2019; 74: 2239-46.

8. Livermore DM, Warner M, Jamrozy D, et al. In Vitro Selection of Ceftazidime-Avibactam Resistance in Enterobacteriaceae with KPC-3 Carbapenemase. Antimicrob Agents Chemother 2015; 59: 5324-30.

9. Barnes MD, Winkler ML, Taracila MA, et al. Klebsiella pneumoniae Carbapenemase-2 (KPC2), Substitutions at Ambler Position Asp179, and Resistance to Ceftazidime-Avibactam: Unique Antibiotic-Resistant Phenotypes Emerge from $\beta$-Lactamase Protein Engineering mBio 2017; 8: e00528-17. 
143 10. Sougakoff W, L'Hermite G, Pernot L, et al. Structure of the imipenem-hydrolyzing class A 144 beta-lactamase SME-1 from Serratia marcescens. Acta Crystallogr D Biol Crystallogr 2002; $145 \quad 58: 267-74$.

146 11. Smith CA, Nossoni Z, Toth $M$, et al. Role of the Conserved Disulfide Bridge in Class A 147 Carbapenemases. J Biol Chem. 2016; 291:22196-206.

148 12. Pettersen EF, Goddard TD, Huang CC, et al. UCSF Chimera-A visualization system for 149 exploratory research and analysis. J Comput Chem 2004; 25: 1605-12.

150 13. Dortet L, Agathine A, Naas T, et al. Evaluation of the RAPIDEC ${ }^{\circledR}$ CARBA NP, the Rapid CARB 151 Screen $^{\circledR}$ and the Carba NP test for biochemical detection of carbapenemase-producing Enterobacteriaceae. J Antimicrob Chemother 2015; 70: 3014-22.

14. Bernabeu S, Dortet $L$, Naas T. Evaluation of the $\beta-C A R B A^{\mathrm{TM}}$ test, a colorimetric test for the rapid detection of carbapenemase activity in Gram-negative bacilli. J Antimicrob Chemother 2017; 72: 1646-58.

15. Dortet L, Tandé $D$, de Briel D, et al. MALDI-TOF for the rapid detection of carbapenemaseproducing Enterobacteriaceae: comparison of the commercialized MBT STAR ${ }^{\circledR}$-Carba IVD Kit with two in-house MALDI-TOF techniques and the RAPIDEC ${ }^{\circledast}$ CARBA NP. J Antimicrob Chemother 2018; 73: 2352-59.

16. Boutal H, Vogel A, Bernabeu S, et al. A multiplex lateral flow immunoassay for the rapid identification of NDM-, KPC-, IMP- and VIM-type and OXA-48-like carbapenemaseproducing Enterobacteriaceae. J Antimicrob Chemother 2018; 73: 909-15. 
Table 1. MIC profiles of E. coli TOP10 expressing single KPC mutants and performances of various diagnostic tests.

\begin{tabular}{|c|c|c|c|c|c|c|c|c|c|c|c|c|c|c|c|c|c|c|c|c|}
\hline \multirow[t]{2}{*}{ AA changes } & \multirow[t]{2}{*}{ Variant } & \multicolumn{11}{|c|}{$\mathrm{MICs}(\mathrm{mg} / \mathrm{L})$} & \multicolumn{4}{|c|}{ Carbapenem hydrolysis assays } & \multicolumn{2}{|c|}{ PCR } & \multicolumn{2}{|r|}{ LFIA $^{a}$} \\
\hline & & AMX & ATM & CRO & CAZ & $\begin{array}{c}\text { CAZ / } \\
\text { AVI }\end{array}$ & CFX & CTX & FEP & IMP & MEM & ERT & $\begin{array}{l}\text { Carba } \\
\text { NP test }\end{array}$ & $\begin{array}{l}\text { RAPIDEC }^{\oplus} \\
\text { CARBA NP }\end{array}$ & $\begin{array}{c}- \\
\text { CARBA }^{\text {TM }}\end{array}$ & $\begin{array}{l}\text { MBT STAR- } \\
\text { Carba IVD }\end{array}$ & $\begin{array}{l}\text { In house } \\
b / a_{\mathrm{KPC}} \mathrm{PCR}\end{array}$ & $\begin{array}{c}\text { Xpert } \\
\text { Carba-R }\end{array}$ & $\begin{array}{l}\text { NG-Test } \\
\text { CARBA } 5\end{array}$ & $\begin{array}{l}\text { Coris Resist-4 } \\
\text { O.K.N.V. K-Set }\end{array}$ \\
\hline & KPC-2 & $>256$ & 32 & 16 & 4 & 0.38 & 4 & 8 & 2 & 8 & 3 & 1 & + & + & + & + & + & + & + & + \\
\hline $\mathrm{H} 272 \mathrm{Y}$ & KPC-3 & $>256$ & $>256$ & 48 & $>256$ & 0.75 & 12 & $>32$ & 6 & 8 & 3 & 1 & + & + & + & + & + & + & + & + \\
\hline P104R & KPC-5 & $>256$ & $>256$ & $>256$ & $>256$ & 0.5 & $>256$ & 8 & 2 & 1 & 0.25 & 0.19 & + & + & + & + & + & + & + & + \\
\hline V240G & KPC-6 & $>256$ & $>256$ & 48 & 32 & 0.75 & 16 & $>32$ & 4 & 4 & 3 & 1 & + & + & + & + & + & + & + & + \\
\hline L168M & KPC-12 & $>256$ & 12 & 6 & 4 & 0.38 & 3 & 4 & 1 & 0.75 & 0.25 & 0.125 & + & + & + & + & + & + & + & + \\
\hline D179Y & KPC-33 & 12 & 0.75 & 1 & $>256$ & 8 & 6 & 1.5 & 1 & 0.25 & 0.032 & 0.008 & - & - & - & - & + & + & - & - \\
\hline H272Y, D179Y & KPC-31 & 6 & 0.75 & 2 & $>256$ & 12 & 4 & 1.5 & 1 & 0.25 & 0.032 & 0.008 & - & - & - & - & + & + & - & - \\
\hline D92G & & $>256$ & 64 & 12 & 4 & 0.25 & 4 & 4 & 2 & 1.5 & 0.5 & 0.38 & + & + & + & + & + & + & + & + \\
\hline W105G & & $>256$ & 24 & 1 & 1 & 0.25 & 2 & 0.75 & 0.25 & 1 & 0.19 & 0.125 & + & + & + & + & + & + & + & + \\
\hline A120L & & $>256$ & 32 & 16 & 3 & 0.38 & 3 & 6 & 2 & 6 & 1.5 & 1.5 & + & + & + & + & + & + & + & + \\
\hline G147K & & $>256$ & 64 & 32 & 6 & 0.38 & 6 & 24 & 6 & 6 & 1 & 0.75 & + & + & + & + & + & + & + & + \\
\hline $\mathrm{C} 238 \mathrm{~S}$ & & $>256$ & 2 & 0.25 & 2 & 0.38 & 1 & 0.25 & 0.19 & 0.25 & 0.047 & 0.008 & - & - & - & - & + & + & + & + \\
\hline V240A & & $>256$ & $>256$ & 64 & 12 & 0.38 & 12 & $>32$ & 4 & 8 & 2 & 1.5 & + & + & + & + & + & + & + & + \\
\hline E. coli TOP10 & & 6 & 0.047 & 0.032 & 0.012 & 0.012 & 0.38 & 0.064 & 0.064 & 0.25 & 0.032 & 0.004 & - & - & - & - & - & - & - & - \\
\hline
\end{tabular}

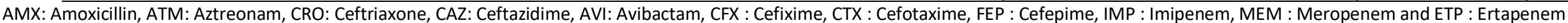

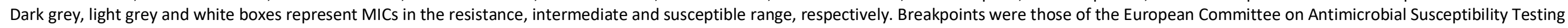

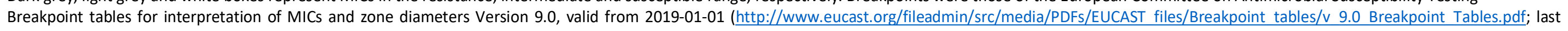
accessed September 2019). Grey boxes represent negative test results.

a LFIA: Lateral flow immunoassay 


\section{Supplementary data}

\section{A}
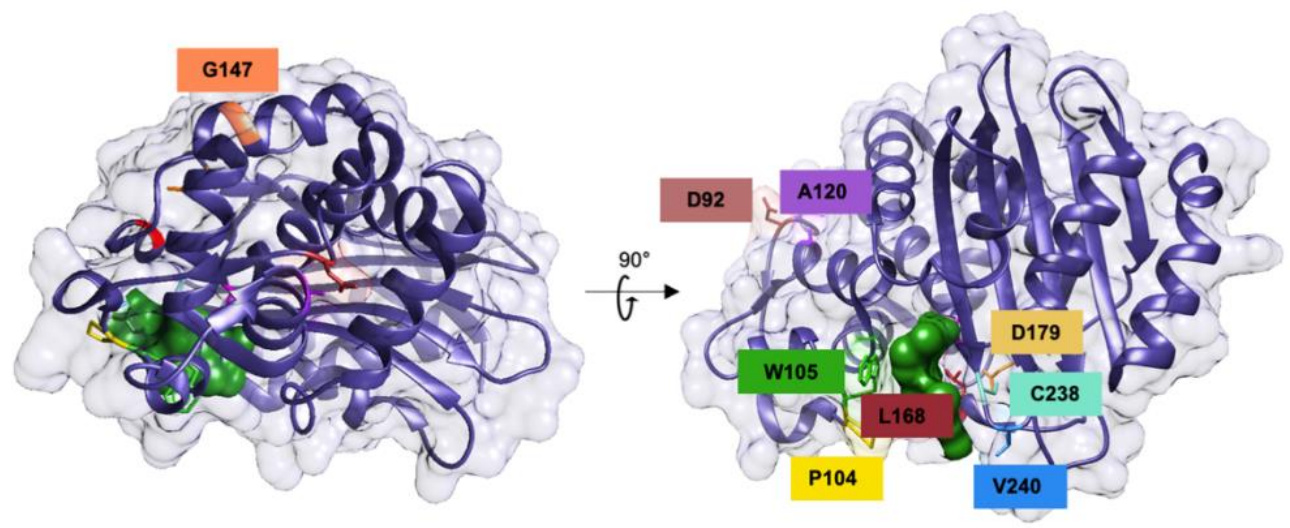

B

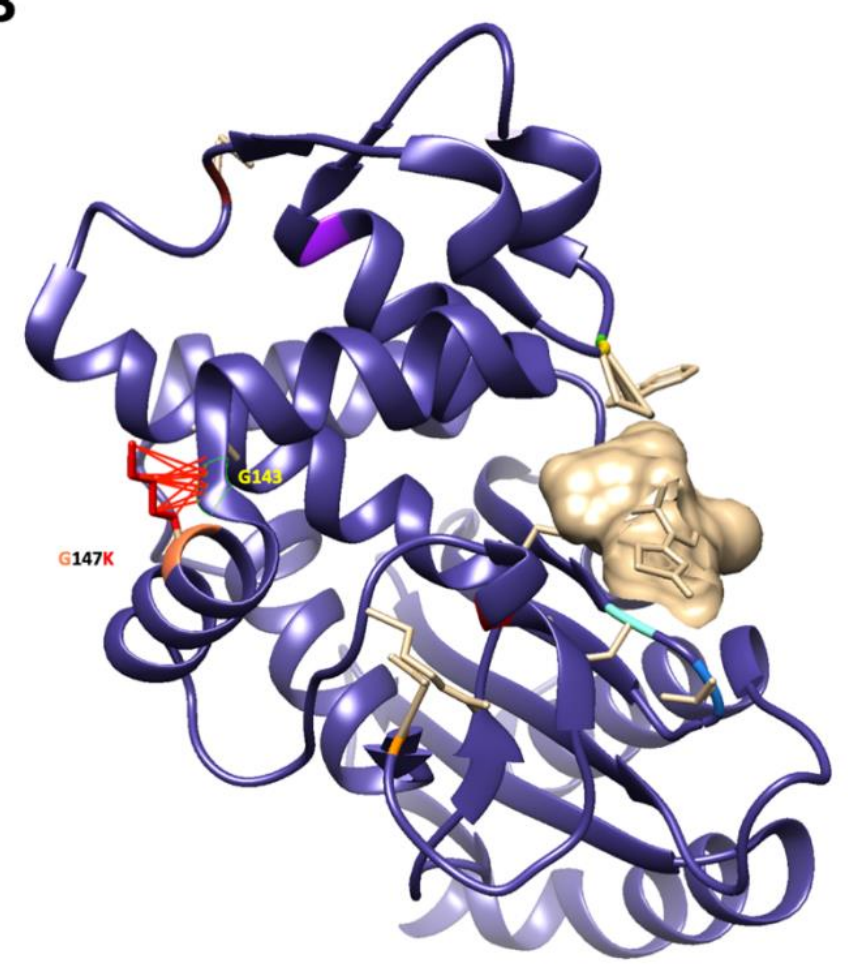

Figure S1. A Positions of the substituted residues included in this study on the Crystal structure of KPC-2 (PDB code 5UJ3) complex with cefotaxime (green), B. Representation of the steric clashes (red lines) between the side chains of K147 and G143. 